\title{
Review of: "Loss of soluble guanylyl cyclase in platelets contributes to atherosclerotic plaque formation and vascular inflammation"
}

\author{
Rory Koenen ${ }^{1}$ \\ 1 Maastricht University
}

Potential competing interests: The author(s) declared that no potential competing interests exist.

In this preprint study by Mauersberger, Sager and colleagues, the role of soluble guanylyl cyclase (sGC) in platelets was investigated in the context of atherosclerosis. This enzyme is part of the nitric oxide (NO) signaling pathway and thus regulates a large variety of NO-controlled processes (e.g. platelet activation and vascular tone). Alleles of the SGC gene were found to influence aspirin therapy for primary prevention of adverse cardiovascular events and the authors aimed to clarify the role of platelet-derived SGC in the development of atherosclerosis in a mouse model. Platelet-specific genetic deletion of sGC ("PIt-sGC-KO") led to an increase of atherosclerotic plaque formation and to an increase in populations of inflammatory cells inside the plaque and in digested aortic tissues. Also, an increased arrest of leukocytes was observed at atherosclerotic predilection sites by intravital microscopy. Incubation of cultured endothelial cells with supernatants of platelets isolated from PIt-sGC-KO mice led to a subsequent increase of leukocyte adhesion, compared with supernatants isolated from control platelets. Profiling of the proteins released by platelets isolated from control and PIt-sGC-KO mice revealed that angiopoietin 1 ("Ang-1") levels were decreased in the supernatant of PIt-sGC-KO platelets. Platelets isolated from individuals homozygous for the risk allele of the human SGC a-subunit gene had a lower release of Ang-1 than platelets isolated from non-risk allele carriers. Bioinformatic analysis revealed that the SGC subunits and Ang-1 were co-expressed in nearly all tissues from patients undergoing bypass surgery. In addition, there appeared to be a positive correlation between Ang-1 and SGC. Treatment of platelets from wildtype mice with the SGC stimulator BAY-747 lead to an enhancement of Ang-1 release and treatment of endothelial cells with the supernatants of activated platelets without or with BAY-747 lead to lower adhesion of neutrophils. Treatment of hyperlipidemic low-density lipoprotein receptor-deficient (LDL-/-) mice with BAY-747 led to a reduced recruitment of adoptively transferred GFP-expressing myeloid cells into their aortas, compared with controls. In addition, LDL-/- mice treated mice developed smaller atherosclerotic plaques, which was paralleled by a lower content of inflammatory cells in the plaque and in digested aortas.

This is an interesting study with clear translational potential. It provides further evidence for the importance of platelets in the development of atherosclerosis. Numerous experiments were soundly performed in the study and although the connection between platelet SGC and Ang-1 release appears 
convincing, the study can further improve when a number of remaining questions are addressed:

- The link between sGC stimulation and the role of platelets (and Ang-1) in the development of atherosclerosis in the BAY-747 (and control)-treated mice could be strengthened. This for example, by determining the plasma levels of Ang-1 in BAY-747 and control mice, or more strongly linking the atherosclerosis studies to the proposed actions of platelet-derived Ang-1 (staining, blockade of Ang-1 or its receptors).

- Provide stronger evidence of the molecular connection of sGC activity and Ang-1 release. Surely, this has been discussed by the authors, but since they obviously have access to powerful bioinformatics tools, its potential has not been fully exhausted. The authors might use network analysis (e.g. with tools like String, PlateletWeb) to identify putative targets. It appears a bit surprising that only 1 factor among the many released by platelets is affected by sGC (-deficiency).

Specific comments or questions:

Introduction- this section can be expanded with some more background on the importance of the NOpathway in platelets and the role of the individual SGC subunits. For the general reader, some examples of traditional risk factors would be useful, as well as a definition of "private" mutations. The overall role of platelets in atherosclerosis is controversial mainly in humans. In mice, there is ample evidence of a (mainly) detrimental role of platelets in atherosclerosis.

Methods - the authors should keep in mind that the PF4 promotor might not be entirely specific for platelets since there is a recently described PF4-expressing subset of resident macrophages (see PubmedID: 32673538).

The high-risk allele of sGC is considered to lower expression. Do the authors expect gene-dosage effects also in mice?

Figure 1: Are there any effects of sex on the extent of atherosclerosis in the examined groups? Platelets (and platelet hyperreactivity) may stimulate atherogenesis particularly during early phases (e.g. PubmedID: 25472975). Do the authors have any information whether this also applies in their mouse model?

Figure 2: The authors should also mention the time of treatment in legend and in the main text (particularly since there is mention of a "very early timepoint", p. 13). In addition, the figures would benefit from a third bar, with control conditions (e.g. supernatant of resting platelets and/or resting endothelial cells). This comment also applies to the data shown in figure 3E and 4B. Is it correct that all platelet activations were triggered by shaking? Please specifically mention manner of 
activation in the legends and in the text describing the experiments (it is mentioned a bit late in the discussion).

Figure 3: Ang-1 release might be expressed relative to the number of platelets in panels $C, D$ and $F$, as was done in panel B. The authors may consider moving panel 3E to the supplement. Tie2 is also expressed on (a subset of) monocytes and macrophages. Do the authors expect a role of Tie2 on these cells as well?

Figure 4: Besides the need of a control bar (e.g. resting platelets in panel 4B), the authors have chosen a different readout ("fold change" vs "RFU" in figure 2). This might be adjusted or explained.

Do the authors have information on the occurrence of platelet-leukocyte complexes in PIt-sGC-KO mice? These complexes are an indicator of platelet activation and platelets also bridge monocytes to the vessel wall, which might provide an explanation for the increased adhesion seen in PIt-sGC-KO mice.

The legends can be a bit more informative with regard to experimental details. 